\title{
EL RECURSO AGUA: ¿FUTURO FOCO DE CONFLICTO EN EL MEDIO ORIENTE?
}

\author{
Frank Tressler Zamorano
}

\begin{abstract}
A menos que se produzcan cambios profundos en la implementación de una política uniforme respecto al agua, el control y la distribución de este recurso podría ser la causa de un nuevo foco de conflicto en el Medio Oriente. Al respecto, es de suma importancia que detrás de la solución a este problema esté el concepto de desarrollo sustentable que necesariamente debe contemplar la protección del medio ambiente. Sin embargo, los proyectos que se están llevando a cabo actualmente no abordan este asunto en forma integral, sino que al contrario han intensificado las diferencias. En este sentido, según el autor, Estados Unidos debe asumir un rol de liderazgo y sugiere algunas soluciones que involucren innovaciones tecnológicas, acciones diplomáticas y participación de organizaciones internacionales afines.
\end{abstract}

\section{I.- Introducción.}

La zona que los países de Occidente llaman Medio Oriente y que a su vez es conocida por varios otros nombres, como la cuna de la civilización, la zona de la media luna y otros más, ha sido foco de atención mundial por diversos motivos. Sin embargo, estas múltiples denominaciones no reconocen la continua característica de conflicto que dicha área ha tenido. Muchas veces el conflicto ha surgido cuando un Estado ha poseído en forma abundante un recurso, de tal manera que otro Estado de la región se siente amenazado y percibe que debe proteger sus intereses en la zona y los recursos que posee en forma escasa. La Guerra del Golfo Pérsico refleja este tipo de conflicto.

A pesar de lo anterior, está naciendo un nuevo tipo de foco de conflicto que constituiría una amenaza más profunda que el control del petróleo. Con la excepción de Turquía, ningún Estado del Medio Oriente posee en forma abundante el recurso del agua. El futuro foco y causa de conflicto será el del control y distribución de dicho recurso, 
a menos que existan cambios radicales en la implementación de una política uniforme respecto del agua.

Es necesario que deba estudiarse la magnitud del problema con el fin de que pueda desarrollarse y llevarse a cabo una política coherente. El tema se divide en dos grandes categorías: la primera, se refiere a las condiciones generales de la región y, la segunda, está constituida por las políticas individuales de los Estados, en orden de mejorar o modificar las condiciones imperantes. Haciendo un análisis del tema, se deben considerar variadas soluciones para diversos problemas, y para ello existen buenos programas y tecnología. Son necesarios una nueva política y un nuevo desarrollo a fin de llevar a cabo una política unificada que sea efectiva. En este sentido, se necesitan potencias líderes como los Estados Unidos, y prueba de ello ha sido el significativo avance del proceso de paz entre Israel y Palestina, gracias al liderazgo que este país ha ejercido.

La solución principal a los problemas contemporáneos del desarrollo sustentable, el crecimiento explosivo de la población, la escasez de los recursos naturales (en especial del vital recurso agua), y las complicaciones políticas regionales, se encuentra antes que nada en la educación dirigida a las personas.

\section{II.- Desarrollo sustentable.}

El desarrollo económico es de primordial importancia para muchos Estados, ya que constituye una opción viable para tener una posición importante en el contexto de las potencias. Cualquier recomendación de política a seguir debe incluir el concepto de desarrollo sustentable. Esto tiene un significado diferente para las naciones del Tercer Mundo y para los países industrializados. Por ejemplo, Turquía, que será estudiada en extensión en este trabajo, se encuentra desarrollando uno de los proyectos más ambiciosos del mundo relacionado con el recurso agua. El Proyecto del Sureste de la Anatolia, conocido como "GAP", tiene su origen en un programa que tiene el objetivo de proveer energía hidroeléctrica y agua para la irrigación de la empobrecida región del Sureste del páís. Desde su inicio, este proyecto se ha convertido en "multisectorial, integrándose con el crecimiento de la región, el cual cubre los aspectos más amplios del desarrollo, incluyendo lo económico, lo social, lo cultural, la infraes- 
tructura y las áreas industriales". ${ }^{1}$ Sin embargo, el desarrollo ambiental se encuentra ausente de la lista de estudios del proyecto. En general, el crecimiento económico a corto plazo no contempla el costo ecológico para los países del Medio Oriente. Turquía y los demás países del Medio Oriente se encuentran activamenté llevando a cabo ambiciosos proyectos de desarrollo del recurso agua, que como resultado exceden significativamente los montos del vital recurso que los ríos Tigris, Eufrates y Jordán pueden proveer. ${ }^{2}$

"GAP" representa un proyecto que enorgullece y da mucha esperanza al pueblo turco. Diseñado y fundado desde el interior de Turquía, la represa Ataturk y la planta de generación hidroeléctrica constituirían la novena represa más alta del mundo y podría entregar energía eléctrica limpia y barata a una extensa y empobrecida zona que limita con Siria e Irak. Este ambicioso proyecto contempla en definitiva la construcción de 22 represas y 19 plantas de generación hidroeléctrica sobre los ríos Tigris y Eufrates, y se espera que la última etapa del proyecto sea finalizada en el año 2005. Las proyecciones del Gobierno de Ankara señalan que 1.7 millones de hectáreas podrán ser irrigadas y sobre $27.000 \mathrm{GWh}$ de energía eléctrica serán generadas anualmente.

El plan contempla una gran expansión en el área de la agricultura, y esto a su vez conlleva un desarrollo de industrias relacionadas con el agro. Parte del proyecto se encuentra en funcionamiento y los resultados ya están a la vista: tierras que antes eran semiáridas ahora están produciendo hasta tres cosechas anuales. ${ }^{3}$ Un cuadro en que se multiplican las áreas de cultivo, se mejora la infraestructura urbana y se desarrollan las industrias relacionadas con el agro como la de pesticidas, herbicidas, plantas de empaque, produciría una tasa anual de crecimiento estimada del $6.8 \%$ en el Producto Nacional Bruto. ${ }^{4}$ Un plan detallado de acción nos muestra un país -Turquía-con población joven, ligada a Europa, y con el control de una región de alto crecimiento industrial. En cierta medida, algunos préstamos de

\footnotetext{
${ }^{1}$ Turquía, Directorio General de Prensa c Información, "GAP: Proyecto del Sureste de la Anatolia", Ankara, Turquía, 1990, p. 2.

${ }^{2}$ Starr, Joyce R. y Daniel C. Stoll, U.S. Foreign Policy on Water Resources in the Middle East, (Washington: CSIS, diciembre, 1987), p. S-11.

${ }^{3}$ Ward, Diane Raines, "In Anatolia, a massive dam project drowns traces of an ancient past", Smithsonian, Washington, agosto 1990, p. 34.

${ }^{4}$ Turquía la Planificación Organizacional del Estado, "Executive Summary" GAP: Southeastem Anatolia Project Master Plan Study, Final Master Plan Report, v. 1, Ankara, Turquía, junio 1990, Cuadro 7.
} 
F. Tressler / El recurso agua: ifuturo foco de conflicto...

Japón están ayudando a materializar este proyecto y con esto a hacer realidad el sueño de muchos.

En el papel, "GAP" aparece como un ambicioso proyecto destinado a elevar a países en vías de desarrollo al rango de países económicamente solventes. Sin embargo, el programa no toma en consideración importantes factores ecológicos y ambientales. Un estudio relacionado con "GAP", denominado "Resumen Ejecutivo del Reporte del Plan Maestro", se refiere al hecho de mantener un sistema ecológico sustentable en relación al crecimiento económico. Este resumen recomendó iniciar un estudio del costo ambiental del proyecto, en orden de "identificar problemas ambientales y formular contramedidas". 5 Finalmente, para efectos de que exista un sistema de desarrollo completo e integrado, es aconsejable un efectivo sistema de monitoreo, control y administración del proyecto en cuestión.

Desafortunadamente, dejando a un lado la breve referencia respecto del costo ecológico del programa de Anatolia, en lo específico del proyecto no se utilizan criterios ambientales para definir los planes y proyecciones. El objetivo del proyecto "GAP" es un intenso desarrollo económico; sin embargo las preocupaciones ecológicas no se encuentran consideradas, con la excepción de una discusión sobre los efectos del proyecto en el abastecimiento del agua a Siria e Irak. ${ }^{6}$ Las mayores áreas de preocupación ecológica se mantienen poco estudiadas o ignoradas. El nervio central del desarrollo agroindustrial lo constituirian las industrias de pesticidas y herbicidas (químicas). A diferencia de sus contrapartes en el Medio Oriente, los agricultores de los países desarrollados han empezado a comprender lo perjudicial que significa la aplicación constante de químicos para la calidad del suelo, su producción y la calidad del agua. El impacto de una gran represa en el ecosistema de la región aún no ha sido analizado, y otras obras de este tipo han demostrado que se inflige un importante daño al ecosistema con proyectos de esta magnitud. Finalmente, el escaso abastecimiento del recurso agua que se produciría con la construcción de represas sucesivas será analizado más adelante en este trabajo.

En otra área, el río Eufrates es el que proporciona la mayor parte del recurso de agua para Siria. Sin incluir las proyecciones de "GAP", varios proyectos bilaterales entre Siria y Turquía en la parte

\footnotetext{
5 Turquía, "State Planning Organization", junio 1990, p. 18.

${ }^{6}$ Inan, Kamram,"Southeastern Anatolia Project and Turkey's Relations with the Middle Eastem Countries", Middle East Business and Banking, marzo 1990, p. 5.
} 
superior del río han reducido la producción agrícola de Siria en un $30 \%$, forzando a racionar el abastecimiento de agua y electricidad en las ciudades. ${ }^{7}$ Siria, en conjunto con Turquía, ha diseñado un Plan de Paz de las Aguas del Eufrates donde el desarrollo es el pilar fundamental. ${ }^{8}$ Debido a diferencias políticas, estos proyectos se encuentran paralizados, pero en caso de materializarse constituirían una seria amenaza para el abastecimiento de agua en Irak, especialmente si se le agrega "GAP".

Según informes turcos, Irak se encuentra abocado en la construcción de una gran infraestructura acuífera destinada a promover el desarrollo de varias industrias agrícolas, de energía bidroeléctrica y de pesca. $^{9}$ Al Norte de Bagdad se ha construido un canal que uniría el río Tigris con el lago Thartar, lo que dará una gran disponibilidad de agua y, si se le agrega una cantidad de represas construidas recientemente, proporcionará a Irak una gran capacidad de reserva. ${ }^{10}$ Nuevamente, la preservación del ecosistema aparece relacionada con la salud pública. Si consideramos que mucha del agua que Irak está utilizando ya ha pasado por los proyectos de desarrollo de Turquía y Siria, estaríamos frente a posibles aguas contaminadas y en un gran porcentaje con desechos químicos (pesticidas y herbicidas).

En otra región, al comparar el río Jordán con otros cursos de agua no se le puede considerar un gran río, ya que la cantidad de agua que anualmente corre por él es de 1.29 millones de metros cúbicos. ${ }^{11}$ Sin embargo, tanto Siria como Israel y Jordania dependen del Jordán y de su mayor tributario, el Yarmuk, para mantener su desarrollo. La necesidad de estas aguas para un desarrollo económico sustentable y las históricas tensiones políticas existentes en la región, conforman un escenario de posibles conflictos en relación a la disputa de los derechos del escaso recurso del agua.

En el caso de Israel, la agricultura juega un papel importante en la rápida expansión de su economía, requiriendo el $75 \%$ del agua que ese país consume anualmente. No existen mayores proyectos a futuro que vayan a demandar un incremento del abastecimiento de dicho recurso. Sin embargo, la agroindustria está creciendo bastante

\footnotetext{
${ }^{7}$ Bakr, Kamran, "Storm over the Euphrates"; World Press Review, mayo 1990, p. 64.

${ }^{8}$ Erengül, Bilge, "GAP and Interregional Trade", Middle East Business and Banking, marzo 1990, p. 12.

${ }^{9}$ Ibid., p. 12.

${ }^{10}$ Ibid, p. 12.

${ }^{11}$ Starr y Stoll, op. cit., p. 5.
} 
rápido y se realizan grandes esfuerzos a fin de proveerla del agua necesaria. Al mismo tiempo, es poco probable que se realicen significativas reducciones de su consumo destinadas a la agricultura. De acuerdo con Stari y Stoll, Israel "consume cinco veces más agua que los países vecinos". 12

En el Reino de Jordania, las corrientes de los ríos Yarmuk y Jordán son esenciales para la subsistencia de sus ciudades y su agricultura. Con el fin de garantizar el suministro en el país, Jordania ha firmado un acuerdo con Siria para construir conjuntamente "La Represa de la Unidad". ${ }^{13}$ Este proyecto también contendría muclios de los problemas ecológicos y ambientales que presenta "GAP".

Con respecto a Siria, a medida que se va materializando " $\mathrm{GAP}^{\text {", }}$ depende más del abastecimiento que le puedan ofrecer los ríos Yarmuk y Jordán. Existe un proyecto para construir "una serie de pequeñas y medianas represas que eventualmente podrían retener hasta el $40 \%$ de las aguas del Yarmuk, en caso que los acuerdos con Jordania no sean respetados" 14 De todas formas, Siria está decidida en mantener un cierto nivel de desarrollo garantizado por un constante abastecimiento de agua.

Cada uno de estos países del Medio Oriente ha determinado que el desarrollo económico sea un objetivo primordial, y el recurso agua sería necesario para alcanzarlo. Las ansias de lograr estas metas traen como consecuencia un olvido de las preocupaciones ambientales por parte de estas naciones. Pesticidas, herbicidas, represas, aumento de la productividad agrícola de los suelos, cultivos intensivos y la destrucción de ecosistemas, son temas que no se encuentran adecuadamente analizados en las políticas de desarrollo de los países mencionados. Si no se analizan los costos ambientales a largo plazo, se crearía una situación de desesperación y de mayor pobreza en un futuro no muy lejano.

La disponibilidad del recurso agua está muy ligada al problema del desarrollo económico que están enfrentando cada uno de los países del Medio Oriente. Tan sólo un Estado en la región -Turquíaincluyendo los países del Golfo Pérsico y Egipto, posee de manera abundante el recurso. Como resultado de un rápido crecimiento económico y de la población en el área, el agua como recurso

\footnotetext{
${ }^{12} \mathrm{Ibid}$, p. 6.

${ }^{13}$ Ibid, p. 7.

${ }^{14}$ Ibid, p. 7.
} 
comienza a ser más escaso. El crecimiento anual de la población en la región es del $2 \%$, en tanto los estudios señalan que cualquier incremento por sobre el $1 \%$ es inmanejable a futuro. ${ }^{15}$ Los recursos escasos de la región se encuentran cada vez más desgastados por este rápido desarrollo y explosivo aumento demográfico.

\section{III.- Conflictos políticos.}

El clima político del Medio Oriente no necesita ser analizado extensamente en este trabajo. La combinación de diferencias políticas y religiosas, conflictos históricos y la falta de cooperación y de confianza entre los Estados, estaría desapareciendo con los primeros esfuerzos de paz llevados a cabo en Washington en 1993. Sin embargo, a pesar de estos acuerdos, todavía falta mucho camino por recorrer para obtener la estabilidad tan anhelada. Se ha creado un primer indicio de cooperación, pero aún falta que transcurra tiempo para borrar las profundas heridas existentes. En cualquier análisis politológico y/o económico que se realice sobre la zona, se debería dar un mayor peso al problema de ecosistema como futuro foco de conflicto.

Uno de los aspectos más destacados en las publicaciones turcas relacionadas con "GAP", es el hecho de que tanto Siria como Irak no serían privados del abastecimiento de agua. Esta postura defensiva se debe a que en el proceso de planificación del proyecto, los países más dependientes del abástecimiento del Tigris y Eufrates no fueron consultados. Existe una comisión trilateral del río Eufrates conformada por Irak, Siria y Turquía. Sin embargo "tan sólo han discutido aspectos técnicos, como la cuantificación de la corriente y la caída de agua lluvia". ${ }^{16}$ Más aún, estudios turcos sobre la proyección de la corriente del río después de terminado el "GAP", señalan que el Eufrates que usualmente lleva 30 billones de metros cúbicos a Irak se vería reducido a 11 billones cuando el proyecto se termine. ${ }^{17}$ Esta situación deja a Irak con un déficit de 2 billones de metros cúbicos de los 13 billones que necesita cada año para efectos de irrigación y sus proyectos de energía hidroeléctrica. ${ }^{18}$ Siria enfrenta similares

\footnotetext{
${ }^{15}$ Ibid, p. 12.

${ }^{16} \mathrm{Ibid}$, p. 14.

${ }^{17}$ Erengïl, op. cit, p. 12.

${ }^{18}$ Ibid, p. 12.
} 
déficits y sus temores no son apaciguados por la teoría turica de que el proyecto "GAP" produciría un efecto positivo de "desparramo hacia abajo" de los beneficios y del desarrollo de la región en los países vecinos como Siria e Irak. Los escenarios de conflicto se incrementan a futuro, sobre todo si la inestabilidad existente en Irak se mantiene en el tiempo.

Finalmente, el proyecto conjunto sirio-jordano, "Represa de la Unidad", está diseñado con el propósito de almacenar las aguas del Yarmuk y ser utilizadas por esos dos países. Los efectos de este proyecto serían muy perjudiciales para Israel y es por esto que ha presionado a fin de paralizarlo por el momento. ${ }^{19} \mathrm{Si}$ dicho proyecto llegara a ser terminado algún día, Israel interpretaría la represa como una amenaza a sus intereses nacionales y, seguramente, respondería de manera proporcional.

\section{IV.- Proposiciones de solución.}

Como hemos señalado anteriormente, existen serios problemas en el Medio Oriente en relación al abastecimiento del recurso agua, y la implementación de ciertos proyectos de infraestructura ayudan a intensificar dichos problemas en ciertas áreas, mientras los solucionan en otras. Existen soluciones e ideas, pero el problema es hacerlas calzar con los intereses de todos los actores. Una adecuada política necesita combinar las innovaciones tecnológicas con voluntad de desarrollar nuevas técnicas y el desarrollo de una conciencia diplomática y popular que vea la importancia de preservar el escaso recurso en la región, el agua.

\section{Innovaciones tecnologicas como fuente de solucion:}

\section{1.- Irrigación por goteo.}

Israel continúa siendo el líder en la región y en el mundo en el desarrollo de formas novedosas de conservar y utilizar el agua. La irrigación por goteo fue iniciada en este país en la década de los sesenta y en la actualidad constituye el $80 \%$ del sistema de irrigación

${ }^{19}$ Ibid, p. 12. 
de sus viñas y hortalizas. Esta tecnología no sólo necesita $1 / 3$ de la cantidad de agua requerida por los sistemas de irrigación convencionales, sino que permite que fertilizantes líquidos sean llevados directamente a las mismas plantas. Este sistema de entrega directa de fertilizante reduce en gran medida el químico requerido en los sistemas de irrigación abierta y de fertilización por vía aérea. to anterior produce plantas más robustas y con eso disminuye la necesidad del uso intensivo de pesticidas. Como se puede deducir, los retornos vienen a compensar los altos costos iniciales de la instalación de este sistema. Hay que considerar, en todo caso, que la irrigación por goteo tiene un alto costo y sólo puede ser utilizado en ciertos tipos de cultivos. La agricultura de extensión que debe ser cosechada con maquinaria pesada se encuentra dentro del tipo de cultivo que no puede usar este sistema, ya que sería destruido en cada cosecha y su alto costo impide el uso a corto plazo. Sin embargo, se han realizado grandes avances en materia de tendido de cañerías, canales y bombas de irrigación y extracción de agua, en el sentido de hacer de la irrigación por goteo una opción ecológica sustentable viable a largo plazo. ${ }^{20}$

\section{2:- Desanilización.}

El concepto se basa en extraer agua dulce de los océanos, idea que ha sido muy popular en el Medio Oriente, en especial en el Reino de Arabia Saudita. Desafortunadamente, la cantidad de energía necesaria para efectos de remover la sal de una cantidad importante de agua de mar conlleva un alto costo, que sólo las naciones más ricas pueden afrontar. En todo caso, se han realizado grandes avances en la utilización de la energía solar en este proceso de desalinización en los últimos años. ${ }^{21}$ Las investigaciones han tenido éxito en el desarrollo de plantas de desalinización en cadena, y se han hecho significativos progresos en el uso comercial de esta agua desalinizada.

\section{3.- $\quad$ Acueducto de la Paz.}

Durante muchos años, Turquía ha estado realizando atractivas propuestas en orden a construir un acueducto que pueda trasladar agua

\footnotetext{
${ }^{20}$ Postel, Sandra, "Water for Agriculture: Facing the Limits", Worldwatch Institute Paper 93, Workwatch Institute, Washington, diciembre, 1984, p. 38-39.

${ }^{21}$ Starr y Stoll, op. cit, p. 22.
} 
desde las provincias turcas del Este hacia los países áridos del Medio Oriente, específicamente Siria, Jordania y los paísès del Golfo Pérsico, incluyendo Arabia Saudita. En una Conferencia llevada acabo en Washington D.C., auspiciada por el Centro de Estudios Internacionales y Estratégicos (CSIS) en junio de 1987, lo primero que Turquía elaboró fue la propuesta de este acueducto: Las diferencias políticas y los extraordinarios costos del proyecto, estimados en 20 billones de dólares, han obstaculizado la concreción de la propuesta. 22

Las innovaciones tecnológicas han significado un alivio temporal a la crisis de abastecimiento del agua en el Medio Oriente. Debido a una falta de cooperación y consenso regional, incluso entre los mismos países árabes, los pasos necesarios para realizar las mejoras necesarias y alcanzar el anhelado desarrollo sustentable se encuentran paralizados. Es de esperar que después de los acuerdos de paz firmados en Washington en 1993, estas naciones se aboquen a la solución de estos problemas, los que pueden llegar a constituir una seria amenaza para el proceso de paz en que se encuentran.

\section{V.- Políticas necesarias para una solución efectiva.}

\section{1.- Rol de liderazgo de los Estados Unidos.}

A la luz de los extensos problemas que tiene el Medio Oriente en materia del recurso agua y la inestabilidad política que ha llevado a una congelación de proyectos de solución con cierta perspectiva, Estados Unidos debería adelantarse y tomar un rol de liderazgo, de manera que se diseñe una política con relación al agua que beneficie a la región. La mejor manera de diseñar esta política sería el establecer tres etapas: primero, se debe implementar la proposición de los autores Starr y Stoll en cuanto a unificar las diversas políticas existentes de los Estados Unidos en el área; segundo, organizar una conferencia mundial para la región que tenga como preocupación el tema del agua; tercero, trabajar con organizaciones internacionales como las Naciones Unidas y sus organismos especializados como el 
Banco Mundial, en orden a evitar que los esfuerzos de la conferencia internacional se pierdan.

\section{2.- Unificar la política norteamericana.}

Muchas agencias federales norteamericanas están preocupadas por el tema de la irrigación y del agua en el Medio Oriente. Al mismo tiempo, sólo el Departamento de Estado tiene la autoridad para negociar internacionalmente con los países del área. ${ }^{23} \mathrm{Con}$ el fin de evitar duplicidad de acciones y tener una sola política para la región, sería necesario la creación de una agencia, bajo el control de la Casa Blanca, para que coordine las diversas instituciones que tratan el tema. Esta nueva agencia de coordinación de las políticas del Medio Oriente, diseñaría políticas a largo piazo, pondría fin a la gran burocracia existente en la toma de decisiones y permitiría la distribución en forma más eficiente de los recursos. Esta visión integral lograría que se tomaran en cuenta de manera más efectiva los costos y aspectos ecológicos. ${ }^{24}$

\section{3.- Conferencia Internacional del Agua.}

Impulsada por las naciones industrializadas, se debería llamar a una Conferencia Mundial del Agua a la que debieran concurrir todos los países, incluso aquéllos conflictivos como Irak. En la agenda de dicha conferencia -la que debiera ser apoyada por los países industrializados- la educación tendría que estar como primera prioridad, con el fin de diseñar políticas a largo plazo.

La destrucción de los ecosistemas debe ser otro punto prioritario de la agenda. Por ejemplo, a Turquía se le debería demostrar las consecuencias a largo plazo que tiene el uso de agroquímicos y los resultados que el reducido caudal de los ríos produce a la biodiversidad respecto de los países ribereños afectados. No sólo habría que educar a los diplomáticos que asisten a dicha Conferencia, sino que también organizar una campaña de educación dirigida a la opinión pública de los países afectados usando los medios de comunicación

${ }^{23}$ Ibid, p. 25.

${ }^{24}$ Gore, Albert, Earth in the Balance: Ecology and the Hurman Spirit, (Houghton Mifflin: 1992), p. 297. 
F. Tressler / El recurso agua: ifuturo foco de conflicto...

social, con el objeto de que adquieran conciencia del problema que les aqueja.

Tendría que planificarse una serie de conferencias multilaterales con el fin de definir una política integral en relación al Tigris/Eufrates, el Yarmuk/Jordán, y el Acueducto de la Paz. Al mismo tiempo, deberían establecerse esquemas que estipulen plazos para implementar innovaciones tecnológicas, señalando las fuentes de financiamiento. En especial se debe estudiar la aplicación de la energía solar, tan abundante en la región, en conjunto con los diversos métodos de irrigación ya analizados. El Banco Mundial, con su departamento del Medio Ambiente Global, podría tener un rol activo, siendo una institución que tiene los medios y la experiencia para afrontar estas situaciones.

\section{4.- Apoyo de las Naciones Unidas.}

Todo diseño de política y desarrollo debe contemplar a las Naciones Unidas, a través del Programa de Naciones Unidas para el Medio Ambiente y el Programa de Naciones Unidas para el Desarrollo. Estos programas pueden servir como control y a su vez como un medio para verificar la correcta implementación de las políticas. Naciones Unidas puede participar en el establecimiento de programas de educación y transferencia tecnológica. Además, en conjunto con las naciones desarrolladas, puede entregar datos proporcionados por satélites, en orden de verificar el impacto al medio ambiente. Los organismos no gubernamentales como "Worldwatch", la Fundación Cousteau y otros, pueden proporcionar importantes consejos sobre estos temas. Esto último es de mucha importancia; debe comenzar a considerarse a estas organizaciones, ya que tienen los profesionales más capacitados y poseen una abundante experiencia sobre los temas ecológicos, pudiendo también influir en la opinión pública para que se vaya formando conciencia del problema.

Como conclusión, teniendo el diseño necesario, es importante no olvidar lo vital que es el liderazgo de los Estados Unidos y el compromiso de ayuda de las demás naciones industrializadas. Esto se ha podido apreciar en las recientes negociaciones de paz, las que concluyeron con la firma de los acuerdos de Washington en 1993. Su éxito se ha debido en gran medida a la presión de los Estados Unidos y a su voluntad política. Según el Embajador Benedick en su libro Ozone Diplomacy, otro ejemplo de logro motivado por el compromi- 
so y el liderazgo ejercido por Estados Unidos fue el Protocolo de Montreal. Además, el buen término en materia de cooperación podría servir de ejemplo para otras regiones con problemas urgentes en materia ambiental, como Sudamérica y Rusia. 